\title{
The Enlargement Rate of Ventricular Septal Rupture: An Overlooked Risk Factor of Delayed Surgery
}

\author{
Fan $\mathrm{Ju}^{1}$, Xin Yuan ${ }^{1}$, Baotong $\mathrm{Li}^{1}$, Xiaokang $\mathrm{Luo}^{1}$, Hengchao $\mathrm{Wu}^{1}$, Tao Yang ${ }^{1}$, and \\ Hansong Sun $^{1}$ \\ ${ }^{1}$ Chinese Academy of Medical Sciences \& Peking Union Medical College Fuwai Hospital
}

September 24, 2021

\begin{abstract}
Objective: The aim of the study was to analyze the impact of rupture size on surgical outcomes of ventricular septal rupture. Methods: During a 15-year period, from Jan 2006 to Dec 2020, 112 patients underwent repairs of postinfarction ventricular septal rupture. Data were collected on clinical, angiographic, and echocardiographic findings; operative procedures; early morbidity and mortality; and survival time. Univariable and multivariable analyses were performed to identify risk factors of 30-day mortality. Results: Thirty-day mortality was $7.1 \%$ for the whole cohort. The mean survival time estimate was 147.2 (95\% $\mathrm{Cl} 135.6-158.9)$ months, with a 3-year survival rate of $91.2 \%$ and a 5-year survival rate of $89.0 \%$. Multivariable analysis regarded rupture enlargement gradient as an independent risk factor of 30-day mortality. The ROC curve indicated that rupture enlargement gradient predicted 30-day mortality with high accuracy. Conclusions: Delayed surgery could be considered for patients who respond well to aggressive treatment. Rupture enlargement gradient is an independent risk factor for postoperative 30-days morality of delayed VSR repair and has good predictive power for the prognosis of VSR patients.
\end{abstract}

\section{INTRODUCTION}

Ventricular septal rupture (VSR) is a rare but fatal mechanical complication of acute myocardial infarction (AMI), with associated medical management mortality that ranges from $41 \%$ to $80 \%^{1-4}$. Traditional opinion believed immediate repair for VSR was necessary because rupture can expand abruptly, resulting in sudden hemodynamic collapse. The 2013 AHA guidelines for STEMI concluded that emergency surgical repair is recommended for all patients with VSR, even in hemodynamically stable patients ${ }^{5}$. Although surgery is recognized as the most effective treatment for VSR, it carries an exceedingly high postoperative mortality of $42.9^{\sim} 80.5 \%$ within 7 days of VSR onset ${ }^{2,6}$.

However, due to an unperfect transportation system in middle-income economies like China, a large proportion of VSR patients were not able to receive emergency transportation to qualified cardiac surgery centers. Therefore, unplanned delayed surgery was more common in China, which differed from the situation in higher-income economies.

On the other hand, delayed surgery has several advantages compared to immediate surgery. Either the difficulty of procedures or the postoperative mortality and morbidity all declined significantly in delayed surgery $^{7}$. As preoperative mechanical assistance got popular, delayed surgery was accepted gradually by some centers ${ }^{8,9}$. The 2017 ESC guidelines for STEMI suggest that delayed surgery could be considered for patients who respond well to aggressive treatment ${ }^{10}$.

Risk factors for postoperative 30-days mortality including advanced age, female gender, renal dysfunction, cardiogenic shock, decreased EF, three-branch lesions, and unstable status requiring preoperative IABP have been identified in the previous researches ${ }^{6}$. Other research found that larger ruptures are more likely to cause preoperative cardiogenic shock ${ }^{7}$, but no direct correlation between rupture size and postoperative mortality 
was reported. However, only a few studies about delayed surgery for VSR were reported, and the majority were case reports and case series. Previous studies left a gap in risk factors analysis of delayed surgery for VSR. In this study, we aim to find the independent risk factors of delayed VSR repair by reviewing the clinical data of VSR patients in our center and analyze the impact of rupture size on the critical preoperative status and surgical outcomes.

\section{MATERIALS AND METHODS}

This was a retrospective study. Ethical approval was obtained from the Fuwai Hospital ethics committee.

\section{Patients}

From Jan 2006 to Dec 2020, 112 consecutive patients with postinfarction VSR underwent VSR repairs and were included in the study with an average follow-up of 65 months. The primary endpoint was postoperative 30-days mortality, and secondary endpoints included critical preoperative status and composite of major adverse cardiovascular events (MACE: cardiac death, myocardial infarction, and cerebral vascular accident ) during follow-up. Clinical and follow-up data were obtained by abstracting the hospital medical records and by phone.

\section{Diagnosis and Definitions}

VSR was defined as (1) the appearance of rough blowing systolic murmurs between the third and fourth left ribs during post-AMI physical examinations; (2) loss of interventricular septum echo on echocardiography, and the presence of a left-to-right shunt; and (3) VSR identified by left ventriculography as indicated by left-to-right shunting of the contrast agent.

Rupture size (Dmax): The preoperative maximum value of defect diameter in mm, measured by transthoracic ultrasound.

Dmin: The preoperative minimum value of defect diameter in $\mathrm{mm}$, measured by transthoracic ultrasound.

Rupture enlargement $(\Delta \mathrm{D}): \Delta \mathrm{D}=\mathrm{Dmax}-\mathrm{Dmin}$

Rupture enlargement rate $(\Delta \mathrm{DR}): \Delta \mathrm{DR}=\Delta \mathrm{D} / \mathrm{T}, \mathrm{T}$ : The minimum time interval between Dmax and Dmin, in days $(\mathrm{d})$.

Critical preoperative status: preoperative severe hemodynamic instability requiring IABP implantation or emergency surgery.

\section{Surgery Strategy}

An individualized delayed surgery strategy was adopted in our center. 1) The hemodynamically unstable patients who showed little response to mechanical assistance would undergo emergency surgery with no hesitation; 2) the patients stabilized by mechanical assistance or vasoactive agent would receive intensive care for at least 28 days before urgent surgery; 3) those stable patients who had not received medicine or mechanical assistance would be monitored strictly in hospital for at least 28 days, and then underwent elective surgery.

In addition to 5 cases of percutaneous septal closure, all the other 107 cases underwent thoracotomy with CPB. Most patients underwent the classical patch procedure. The left ventricle was incised parallel to 1 or 2 $\mathrm{cm}$ away from the anterior or posterior descending artery. Polyester patches were sutured to the uninfarcted or fibrotic ventricular septal tissue, as well as to the uninfarcted ventricle anterolateral wall tissue to isolate the infarcted myocardium from the left ventricle. The left ventricle incision was closed by the felt Sandwich method. For details, refer to a previous report ${ }^{11}$.

\section{Statistical Analysis}

Statistical analysis was performed with IBM SPSS 24.0 software. Statistical results are presented as median (Q1-Q3) or mean \pm standard deviation. Baseline analysis among groups was performed with chi-square tests 
or Fisher's exact tests for categorical data and the 2-tailed t test or the Mann-Whitney test for continuous data. Multivariable analysis was performed for variables that demonstrated statistical significance in the univariable analysis. Logistics regression was used for both univariate and multivariable analysis. Receiver operator characteristic curves (ROC curves) were used for the determination of the best cutoff point in predicting postoperative mortality. This cutoff point was determined by maximizing the Youden index $(=$ sensitivity+specificity-1) of a ROC curve. Survival curves were plotted using the Kaplan-Meier method, and statistical differences were compared using a log-rank test. A P value less than 0.05 was considered statistically significant.

\section{RESULTS}

\section{Baseline Characteristic}

A total of 112 cases were included in the study. (Table 1) There were 66 males and 46 females, with a mean age of $63.0 \pm 8.2$ years. The medical history revealed hypertension in 68 patients $(60.1 \%)$, smoking history in $45(40.2 \%)$, and diabetes mellitus in $39(34.8 \%)$. The site of the infarction was anterior in 86 patients $(76.8 \%)$ and posterior in 26 patients $(23.2 \%)$. The ruptures located at anterior septum in 8 patients $(7.1 \%)$, posterior septum in 23 patients $(20.5 \%)$, and cardiac apex in 81 patients $(72.3 \%)$. (Table 2 )

Thrombolysis was performed in 15 cases (13.4\%), and acute PCI was performed in 30 cases (26.8\%). Time from AMI to the onset of VSR was a median of 4 days, while the time from VSR to surgical intervention was a median of 53.5 days. Acute renal injury was present in 22 patients defined as creatinine value greater than $200 \mathrm{mmol} / \mathrm{L}$. None of the patients had a preoperative chronic renal failure that could confound this definition. All patients except 5 patients had significant coronary lesions revealed by angiography. For the whole cohort, the frequency of 1-, 2-, and 3-vessel disease was not different.

\section{Early Morbidity}

44 patients (39.3\%) required IABP to exit the operating room, while three patients $(2.7 \%)$ need intraoperative implantation of ventricular assist devices. Reoperation of deep sternal infection was performed in six patients (5.4\%). Ten patients (8.9\%) received continuous renal replacement therapy due to postoperative renal failure. Postoperative shunts were found in eleven patients (9.8\%) by postoperative echocardiography, and only one patient underwent reoperation within 30 days after the primary procedure due to severe unstable circulation status.

\section{Thirty-day Mortality}

Thirty-day mortality was $7.1 \%$ for the whole cohort, while $100 \%$ for the acute phase $\left(<7\right.$ days after AMI $\left.{ }^{12}\right)$, $21.4 \%$ for the healing phase (7-28 days), and $4.08 \%$ for the healed phase (29 days and beyond). Significant risk factors of 30-day mortality found by the univariable analysis were age, gender, infarction-surgery interval, previous cerebrovascular accident, $\Delta \mathrm{D}$, and $\Delta \mathrm{DR}$. (Table 3) Independent risk factors of 30-day mortality found by multivariable analysis corrected by 1,000 bootstrap replicates were female gender, elder age, shorter infarction-surgery interval, previous cerebrovascular accident, lower $\Delta \mathrm{D}$, and higher $\Delta \mathrm{DR}$. (Table 4 )

ROC curves for Dmax and $\triangle \mathrm{DR}$ were plotted separately to evaluate their predictive power for 30-day mortality, yielding an area under the curve (AUC) of 0.641 and 0.863 respectively. (Figure 1) The results indicated that $\triangle \mathrm{DR}$ predicted 30-day mortality with high accuracy.

The cut-off point was picked at $0.205 \mathrm{~mm} / \mathrm{d}$ by calculating the maximum of the Youden index. According to the cut-off point, the patients were divided into the low $\triangle \mathrm{DR}$ group and the high $\Delta \mathrm{DR}$ group. The differences in preoperative IABP implantation, EuroSCORE, preoperative critical status, CPB time, aortic clamp time, postoperative ventilation time, ICU stay, reoperation, and 30-day mortality were statistically significant between the two groups. (Table 5)

\section{Mid-term Survival}

Ninety-one patients were included in the follow-up, and the other 21 patients were lost during follow-up 
because of changing contacts. Six patients died due to MACE during follow-up. The overall median follow-up time was 65 months (IQR 28-101 months). The mean survival time estimate was 147.2 (95\% Cl 135.6-158.9) months, with a 3 -year survival rate of $91.2 \%$ and a 5 -year survival rate of $89.0 \%$.

After grouping, the median survival time estimates for the low $\Delta \mathrm{DR}$ group and the high $\Delta \mathrm{DR}$ group were 154.9 (95\% Cl 143.9-166.6) months and 113.8 (95\% Cl 88.9-138.6) months respectively. Kaplan-Meier overall survival curves and number at risk table for patients with low $\Delta \mathrm{DR}$ group and high $\Delta \mathrm{DR}$ showed a statistically significant difference (Log-rank chi-square 5.775; $\mathrm{p}=0.016)$.(Figure 2 )

\section{COMMENTS}

Postinfarction VSD was an uncommon but challenging mechanical complication for surgeons. In the reperfusion era, approximately $0.2 \%$ of the STEMI patients would be diagnosed as VSR ${ }^{2,13-15}$. However, the reported postoperative mortality of emergency VSR repairs was no less than $30 \%^{1,16-19}$, which nearly stood for the poorest prognosis in cardiac surgery. Therefore, it is important to identify patients with excessive risk in whom surgical intervention should be cautious.

It has been conventionally believed that the average time between infarction and VSR decreased from 5 days to close to 1 day after the introduction of thrombolytic therapy ${ }^{2,20-22}$. However, such a change was not supported by our finding, probably because only $13.4 \%$ of the patients in this research got thrombolysis, and $26.8 \%$ underwent PCI. It is obvious that reperfusion therapy prevents the extensive myocardial necrosis typically associated with mechanical complications ${ }^{2}$. However, patients who already have VSR would benefit little from reperfusion therapy, because their unstable status was related to left-to-right shunt more than myocardial ischemia. So facing unstable VSR patients, many cardiologists preferred IABP implantation rather than PCI or thrombolysis. Similar results were also reported in previous researches ${ }^{6,7,23}$, which support our findings.

In this study, a shorter infarction-surgery interval was found to be a significant risk factor for postoperative mortality, with operative mortality rates of $100 \%, 21.4 \%$, and $4.08 \%$ in the acute, healing, and healed phases, respectively. The result was generally consistent with the previous studies ${ }^{6,24}$. The mortality of emergency VSR surgery kept high due to the hemodynamic instability of patients in the acute phase of AMI, the fragile tissues surrounding the VSR, and hypoperfusion of systemic organs ${ }^{20,24,25}$. However, as mechanical assist implantation has become increasingly popular over the last decades, quite a few patients can be stabilized by mechanical assistance and safely past the acute phase. According to the STS database, patients who underwent surgery within 7 days of presentation had a $54.1 \%$ mortality compared with $18.4 \%$ mortality if the repairs was delayed until after 7 days $^{6}$. With longer infarction-surgery intervals, consequent myocardial fibrosis would significantly reduce the difficulty of surgical procedures, which resulted in good surgical outcomes. For the patients who could be stabilized by mechanical assistance, they would benefit from the delayed surgery. Therefore, the 2017 ESC guidelines for STEMI suggest that delayed surgery could be considered for patients who respond well to aggressive treatment ${ }^{10}$, which agreed with our opinion.

Moreover, the delayed surgery strategy was adopted in our center, not only because of the substantial impact of short infarction-surgery intervals on the surgical outcomes ${ }^{7}$, but also only 22 cases (19.6\%) were transported to our center within 7 days after infarction. The other 90 cases $(80.4 \%)$ already missed the acute phase when they came.

Elder age and female gender were predictors of 30-day mortality in our study, which was also supported with other reports ${ }^{2,6,20}$. Although several reports had different results ${ }^{23}$, it was undebatable that elder or female patients were more vulnerable especially with a risky disease like VSR. Different results were probably due to varied samples.

This study found that the rupture enlargement rate was not only an independent risk factor, but also a strong predictor for postoperative mortality. Moreover, the results showed that the rupture enlargement was related to the critical preoperative status, as well as prolonged postoperative ventilation time, ICU length of stay, and reoperation. Such results were not reported by other researchers. The rupture enlargement rate was 
overlooked in previous studies, probably because it was difficult to observe in the immediate surgery strategy. The VSR patients in previous studies received emergency operations early, so there was not enough time for surgeons to observe the change of rupture size preoperatively. In clinical, the abrupt enlargement of rupture was usually associated with reinfarction or reperfusion injury. After the rupture enlargement, significantly increased shunts and delay in fibrosis at defect edges would lead to hemodynamic instability, sooner timing of surgery, and increased difficulty of operation. These factors result in worse surgical outcomes including increased postoperative mortality and morbidity.

Surprisingly, rupture enlargement was regarded as a protective factor by multivariable analysis with an OR value of 0.464 , which was against the clinical experience. The unstable patients got surgery sooner than the stable patients, so they got less time for rupture to expand despite their higher rupture enlargement rate. In contrast, the stable patients had more time for adjustment before surgery, so they got larger rupture enlargement although their rupture expanded slowly. As a result, the effect of rupture enlargement was corrected in multivariable analysis. Due to the small sample, our results only generated a research hypothesis that needs to be verified in further studies.

In addition, the results showed a statistical significance in rupture size between the critical and noncritical groups, but similar results were not found between the survivors and non-survivors groups. Univariate regression also did not find an association between rupture size and postoperative mortality, and the ROC curve confirmed the poor predictive power of rupture size for postoperative mortality. The conventional opinion believed that a larger rupture size or larger preoperative shunt can lead to cardiogenic shock ${ }^{7,26}$, and it was also verified by our data. The reason that rupture size affects the hemodynamic status was probably that rapid rupture enlargement leads to both bigger rupture size and sudden rise of shunts. However, most of the patients in this study with delayed surgery received preoperative mechanical assistance for weeks, so hemodynamic instability caused by large rupture was likely to be corrected before the operations. Therefore, the impact of rupture size on surgical outcome was eliminated by mechanical assistance in the delayed surgery strategy.

Although the ROC curves demonstrated the satisfactory predictive power of rupture enlargement rate on postoperative mortality, the median values of rupture enlargement rate for the non-survivor and survivor groups were $0.07 \mathrm{~mm} / \mathrm{d}$ and $0.32 \mathrm{~mm} / \mathrm{d}$ respectively, and the cutoff point of the ROC curve was 0.205 $\mathrm{mm} / \mathrm{d}$, which is apparently extremely difficult for transthoracic echocardiography to observe. More accurate examinations and further researches are needed to explain the clinical significance of the rupture enlargement rate.

There are some limitations in this study. The retrospective design of this study makes it more prone to confounding, selection bias, and information bias. The small sample size forced researchers to use Bootstrap in multivariable analysis to correct the result, which led to limited reliability of the results. Multi-center research with large sample size is needed to further confirm the results.

\section{CONCLUSIONS}

Delayed surgery could be considered for patients who respond well to aggressive treatment. Rupture enlargement rate is an independent risk factor for postoperative 30-days morality of delayed VSR repair and has good predictive power for the prognosis of VSR patients. More accurate examinations and further researches are needed to explain the clinical significance of the rupture enlargement rate.

\section{References}

1 Moreyra, A. E. et al. Trends in incidence and mortality rates of ventricular septal rupture during acute myocardial infarction.Am J Cardiol 106 , 1095-1100, doi:10.1016/j.amjcard.2010.06.013 (2010).

2 Crenshaw, B. S. et al. Risk factors, angiographic patterns, and outcomes in patients with ventricular septal defect complicating acute myocardial infarction. GUSTO-I (Global Utilization of Streptokinase and TPA for Occluded Coronary Arteries) Trial Investigators. Circulation 101, 27-32, doi:10.1161/01.cir.101.1.27 (2000). 
3 López-Sendón, J. et al. Factors related to heart rupture in acute coronary syndromes in the Global Registry of Acute Coronary Events. Eur Heart J 31 , 1449-1456, doi:10.1093/eurheartj/ehq061 (2010).

4 French, J. K. et al. Mechanical complications after percutaneous coronary intervention in ST-elevation myocardial infarction (from APEX-AMI). Am J Cardiol 105, 59-63, doi:10.1016/j.amjcard.2009.08.653 (2010).

5 O'Gara, P. T. et al. 2013 ACCF/AHA guideline for the management of ST-elevation myocardial infarction: executive summary: a report of the American College of Cardiology Foundation/American Heart Association Task Force on Practice Guidelines. Circulation 127 , 529-555, doi:10.1161/CIR.0b013e3182742c84 (2013).

6 Arnaoutakis, G. J. et al. Surgical repair of ventricular septal defect after myocardial infarction: outcomes from the Society of Thoracic Surgeons National Database. Ann Thorac Surg 94 , 436-443; discussion 443-434, doi:10.1016/j.athoracsur.2012.04.020 (2012).

7 Li, H. et al. Profile and Outcomes of Surgical Treatment for Ventricular Septal Rupture in Patients With Shock. Ann Thorac Surg 108, 1127-1132, doi:10.1016/j.athoracsur.2019.03.101 (2019).

8 Morimura, H. \& Tabata, M. Delayed surgery after mechanical circulatory support for ventricular septal rupture with cardiogenic shock. Interact Cardiovasc Thorac Surg 31 , 868-873, doi:10.1093/icvts/ivaa185 (2020).

9 Furui, M., Yoshida, T., Kakii, B., Uchino, G. \& Nishioka, H. Strategy of delayed surgery for ventricular septal perforation after acute myocardial infarction. J Cardiol 71 , 488-493, doi:10.1016/j.jjcc.2017.10.016 (2018).

10 Ibanez, B. et al. 2017 ESC Guidelines for the management of acute myocardial infarction in patients presenting with ST-segment elevation: The Task Force for the management of acute myocardial infarction in patients presenting with ST-segment elevation of the European Society of Cardiology (ESC). Eur Heart J 39 , 119-177, doi:10.1093/eurheartj/ehx393 (2018).

11 David, T. E., Dale, L. \& Sun, Z. Postinfarction ventricular septal rupture: repair by endocardial patch with infarct exclusion. J Thorac Cardiovasc Surg 110, 1315-1322, doi:10.1016/s0022-5223(95)70054-4 (1995).

12 Thygesen, K., Alpert, J. S. \& White, H. D. Universal definition of myocardial infarction. J Am Coll Cardiol 50 , 2173-2195, doi:10.1016/j.jacc.2007.09.011 (2007).

13 Harikrishnan, S. et al. Ventricular septal rupture following myocardial infarction: clinical, haemodynamic, angiographic profile and long-term outcome. Int J Cardiol 120, 279-280, doi:10.1016/j.ijcard.2006.07.199 (2007).

14 Nakatani, D. et al. Effect of successful late reperfusion by primary coronary angioplasty on mechanical complications of acute myocardial infarction. Am J Cardiol 92, 785-788, doi:10.1016/s0002-9149(03)00883-x (2003).

15 Yip, H. K. et al. The potential impact of primary percutaneous coronary intervention on ventricular septal rupture complicating acute myocardial infarction. Chest 125 , 1622-1628, doi:10.1378/chest.125.5.1622 (2004).

16 Poulsen, S. H. et al. Ventricular septal rupture complicating acute myocardial infarction: clinical characteristics and contemporary outcome. Ann Thorac Surg 85, 1591-1596, doi:10.1016/j.athoracsur.2008.01.010 (2008).

17 Deja, M. A. et al. Post infarction ventricular septal defect - can we do better? Eur J Cardiothorac Surg 18 , 194-201, doi:10.1016/s1010-7940(00)00482-6 (2000).

18 Jeppsson, A., Liden, H., Johnsson, P., Hartford, M. \& Rådegran, K. Surgical repair of post infarction ventricular septal defects: a national experience. Eur J Cardiothorac Surg 27 , 216-221, doi:10.1016/j.ejcts.2004.10.037 (2005). 
19 Mantovani, V., Mariscalco, G., Leva, C., Blanzola, C. \& Sala, A. Surgical repair of post-infarction ventricular septal defect: 19 years of experience. Int J Cardiol 108, 202-206, doi:10.1016/j.ijcard.2005.05.007 (2006).

20 Menon, V. et al. Outcome and profile of ventricular septal rupture with cardiogenic shock after myocardial infarction: a report from the SHOCK Trial Registry. SHould we emergently revascularize Occluded Coronaries in cardiogenic shocK? J Am Coll Cardiol36 , 1110-1116, doi:10.1016/s0735-1097(00)00878-0 (2000).

21 Birnbaum, Y., Fishbein, M. C., Blanche, C. \& Siegel, R. J. Ventricular septal rupture after acute myocardial infarction. N Engl J Med 347, 1426-1432, doi:10.1056/NEJMra020228 (2002).

22 Murday, A. Optimal management of acute ventricular septal rupture.Heart 89 , 1462-1466, doi:10.1136/heart.89.12.1462 (2003).

23 Lundblad, R., Abdelnoor, M., Geiran, O. R. \& Svennevig, J. L. Surgical repair of postinfarction ventricular septal rupture: risk factors of early and late death. J Thorac Cardiovasc Surg137 , 862-868, doi:10.1016/j.jtcvs.2008.09.008 (2009).

24 Labrousse, L. et al. Surgery for post infarction ventricular septal defect (VSD): risk factors for hospital death and long term results. Eur J Cardiothorac Surg 21, 725-731; discussion 731-722, doi:10.1016/s10107940(02)00054-4 (2002).

25 Rob, D. et al. A rationale for early extracorporeal membrane oxygenation in patients with postinfarction ventricular septal rupture complicated by cardiogenic shock. Eur J Heart Fail 19 Suppl 2, 97-103, doi:10.1002/ejhf.852 (2017).

26 Jones, B. M. et al. Ventricular septal rupture complicating acute myocardial infarction: a contemporary review. Eur Heart J35 , 2060-2068, doi:10.1093/eurheartj/ehu248 (2014).

TABLE 1. Patient data and risk factors of 30-day mortality

\author{
Variable \\ Demographics \\ Age (y) \\ $>=60$ \\ BMI \\ Male Gender \\ Co-morbidities \\ Smoking History \\ Alcohol drinking history \\ Hypertension \\ Diabetes \\ Chronic Obstructive Pulmonary Disease \\ Previous Cerebrovascular accident \\ Serum Creatinine $>200$ \\ Arrhythmia \\ Atrial Fibrillation \\ Ventricular Tachycardia \\ Atrioventricular Block \\ Acuity \\ AMI-VSR time interval (d) \\ AMI-Hospital time interval (d) \\ AMI-Surgery time interval (d) \\ Acute Phase $(<=7 \mathrm{~d})$ \\ Healing Phase $\left(8^{\sim} 28 \mathrm{~d}\right)$
}


Healed Phase ( $>28 \mathrm{~d})$

Thrombolysis

PCI

Vasoconstrictor

Preoperative IABP

Preoperative Mechanical Ventilation

Critical Preoperative Status

EuroSCORE

Angiography

Normal

One-branch lesions

Two-branch lesions

Three-branch lesions

Left main+two-branch lesions

Left main+three-branch lesions

Echocardiography

Location of MI

Anterior wall

Posterior Wall or Inferior Wall

Combined with Ventricular Aneurysm

Location of Rupture

Anterior Septum

Posterior Septum

Cardiac Apex

Rupture Size

Rupture Enlargement

Rupture Enlargement Rate

LVEF

Surgical Status

Emergency Operation

Uegent Operation

Elective Operation

Operative Characteristics

Repair Method

Patch

Direct Suture

Exclusion

Percutaneous Closure

Cardiopulmonary Bypass Time (min)

Aortic Clamping Time (min)

Concomitant CABG

Concomitant Mitral Repair

Postoperative Outcomes

Postoperative Ventilation Time (h)

ICU Stay (d)

Postoperative IABP Time

Continuous Renal Replacement Therapy

Reoperation

Postoperative Shunt

Postoperative Cerebrovascular accident

Postoperative Death 
Values are presented as means $\pm \mathrm{SD}$ or the number of patients or fractions of patients. BMI, body mass index; AMI, acute $\mathrm{n}$

TABLE 2. Clinical Characteristics According to Location of Rupture

\begin{tabular}{|c|c|c|c|c|}
\hline Variable & Anterior Septum $(\mathrm{n}=8)$ & Posterior Septum $(\mathrm{n}=23)$ & Cardiac Apex $(\mathrm{n}=81)$ & $\mathrm{P}$ \\
\hline \multicolumn{5}{|l|}{ Acuity } \\
\hline AMI-VSR time interval (d) & $7.00 \pm 4.44$ & $6.00 \pm 6.88$ & $6.38 \pm 6.17$ & \\
\hline Critical Preoperative Status & $5(62.50 \%)$ & $11(47.83 \%)$ & $33(40.74 \%)$ & \\
\hline EuroSCORE & $15.62 \pm 4.50$ & $12.26 \pm 4.47$ & $13.22 \pm 4.62$ & \\
\hline \multicolumn{5}{|l|}{ Echocardiography } \\
\hline \multicolumn{4}{|l|}{ Location of MI } & \\
\hline Anterior wall & $8(100.00 \%)$ & $5(21.74 \%)$ & $73(90.12 \%)$ & \\
\hline Posterior Wall or Inferior Wall & $0(0.00 \%)$ & $18(78.26 \%)$ & $8(9.88 \%)$ & \\
\hline Combined with Ventricular Aneurysm & $5(62.50 \%)$ & $14(60.87 \%)$ & $60(74.07 \%)$ & \\
\hline Rupture Size & $14.38 \pm 7.39$ & $15.43 \pm 6.06$ & $12.44 \pm 4.28$ & \\
\hline \multicolumn{5}{|l|}{ Operative Characteristics } \\
\hline Repair Method & & & & \\
\hline Patch & $8(100.00 \%)$ & $14(60.87 \%)$ & $58(71.60 \%)$ & \\
\hline Direct Suture & $0(0.00 \%)$ & $5(21.74 \%)$ & $12(14.81 \%)$ & \\
\hline Exclusion & $0(0.00 \%)$ & $4(17.39 \%)$ & $6(7.41 \%)$ & \\
\hline Percutaneous Closure & $0(0.00 \%)$ & $0(0.00 \%)$ & $5(6.17 \%)$ & \\
\hline Cardiopulmonary Bypass Time & $136.88 \pm 31.02$ & $130.57 \pm 51.77$ & $107.91 \pm 58.06$ & \\
\hline Aortic Clamping Time & $85.50 \pm 25.81$ & $96.83 \pm 42.87$ & $71.31 \pm 34.15$ & \\
\hline Concomitant CABG & $3(37.50 \%)$ & $17(73.91 \%)$ & $46(56.79 \%)$ & \\
\hline Concomitant Mitral Repair & $0(0.00 \%)$ & $4(17.39 \%)$ & $1(1.23 \%)$ & \\
\hline \multicolumn{5}{|l|}{ Postoperative Outcomes } \\
\hline Postoperative Shunt & $1(12.50 \%)$ & $2(8.70 \%)$ & $8(9.88 \%)$ & \\
\hline Postoperative Death & $1(12.50 \%)$ & $0(0.00 \%)$ & $7(8.64 \%)$ & \\
\hline
\end{tabular}

TABLE 3. Univariable Analysis for 30-Day Mortality by Logistic Regression Analysis

\begin{tabular}{ll}
\hline & B \\
Male Gender & -2.46 \\
Age & 0.29 \\
BMI & 0.20 \\
Smoking History & -1.64 \\
Alcohol drinking history & -0.57 \\
Hypertension & 0.71 \\
Diabetes & 1.23 \\
AMI-surgery time interval (d) & -0.08 \\
Thrombolysis & -18.79 \\
PCI & -1.00 \\
Chronic Obstructive Pulmonary Disease & -18.65 \\
Previous Cerebrovascular accident & 2.71 \\
Atrial Fibrillation & 1.57 \\
Ventricular Tachycardia & -18.66 \\
Vasoconstrictor & 19.14 \\
Preoperative IABP & 19.64 \\
Ccr & -0.04 \\
Surgical Status &
\end{tabular}


Location of MI $\quad 0.80$

$\begin{array}{ll}\text { LVA size } & 0.00\end{array}$

Rupture Size $\quad 0.04$

Rupture Enlargement $\quad 0.14$

Rupture Enlargement Rate $\quad 1.58$

$\begin{array}{ll}\text { LVEF } & -0.01\end{array}$

Ccr, creatinine clearance rate; LVA, left ventricular aneurysm. Ccr, creatinine clearance rate; LVA, left ventricular aneury

TABLE 4. Univariable Analysis for 30-Day Mortality by Logistic Regression Analysis

Rupture Enlargement Rate

Rupture Enlargement

Age

Male Gender

AMI-surgery time interval

Previous Cerebrovascular accident

$\beta$, Regression coefficient; OR, odds ratio; $95 \%$ CI, 95\% confidence interval. Bootstrap was conducted with 1,000 replicates.

ТАВ

\begin{tabular}{|c|c|c|c|}
\hline Variable & Low $\Delta$ DR group $(\mathrm{n}=80)$ & High $\Delta \mathrm{DR}$ group $(\mathrm{n}=32)$ & $\bar{P}$ Value \\
\hline \multicolumn{4}{|c|}{ 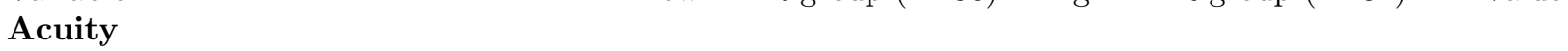 } \\
\hline AMI-VSR time interval (d) & $6.54 \pm 6.43$ & $5.94 \pm 5.52$ & 0.646 \\
\hline AMI-Hosp time interval (d) & $78.78 \pm 206.45$ & $24.16 \pm 30.57$ & $<0.001$ \\
\hline AMI-surgery time interval (d) & $105.39 \pm 210.47$ & $47.56 \pm 38.58$ & $<0.001$ \\
\hline Acute Phase $(<=7 \mathrm{~d})$ & $0(0.00 \%)$ & $1(3.12 \%)$ & \\
\hline Healing Phase ( $\left.8^{\sim} 28 \mathrm{~d}\right)$ & $6(7.50 \%)$ & $8(25.00 \%)$ & \\
\hline Healed Phase $(>28 \mathrm{~d})$ & $74(92.50 \%)$ & $23(71.88 \%)$ & \\
\hline Critical Preoperative Status & $27(33.75 \%)$ & $22(68.75 \%)$ & $<0.001$ \\
\hline EuroSCORE & $12.15 \pm 4.28$ & $15.81 \pm 4.40$ & $<0.001$ \\
\hline \multicolumn{4}{|l|}{ Echocardiography } \\
\hline Rupture Size & $11.50 \pm 3.82$ & $17.44 \pm 5.30$ & $<0.001$ \\
\hline Rupture Enlargement Rate & $0.06 \pm 0.07$ & $0.56 \pm 0.53$ & $<0.001$ \\
\hline LVEF & $49.79 \pm 10.05$ & $48.06 \pm 10.41$ & 0.418 \\
\hline Surgical Status & & & 0.001 \\
\hline Emergency Operation & $6(7.50 \%)$ & $10(31.25 \%)$ & \\
\hline Uegent Operation & $40(50.00 \%)$ & $17(53.12 \%)$ & \\
\hline Elective Operation & $34(42.50 \%)$ & $5(15.62 \%)$ & \\
\hline \multicolumn{4}{|l|}{ Operative Characteristics } \\
\hline Cardiopulmonary Bypass Time & $108.30 \pm 54.63$ & $130.47 \pm 57.31$ & 0.058 \\
\hline Aortic Clamping Time & $70.44 \pm 31.04$ & $95.38 \pm 44.08$ & $<0.001$ \\
\hline \multicolumn{4}{|l|}{ Postoperative Outcomes } \\
\hline Postoperative Ventilation Time & $47.85 \pm 74.85$ & $86.19 \pm 132.50$ & 0.021 \\
\hline ICU Stay & $5.94 \pm 5.90$ & $9.47 \pm 8.85$ & 0.003 \\
\hline Continuous Renal Replacement Therapy & $5(6.25 \%)$ & $5(15.62 \%)$ & 0.116 \\
\hline Reoperation & $2(2.50 \%)$ & $4(12.50 \%)$ & 0.034 \\
\hline Postoperative Shunt & $8(10.00 \%)$ & $3(9.38 \%)$ & 0.92 \\
\hline Postoperative Cerebrovascular accident & $0(0.00 \%)$ & $1(3.12 \%)$ & 0.112 \\
\hline
\end{tabular}



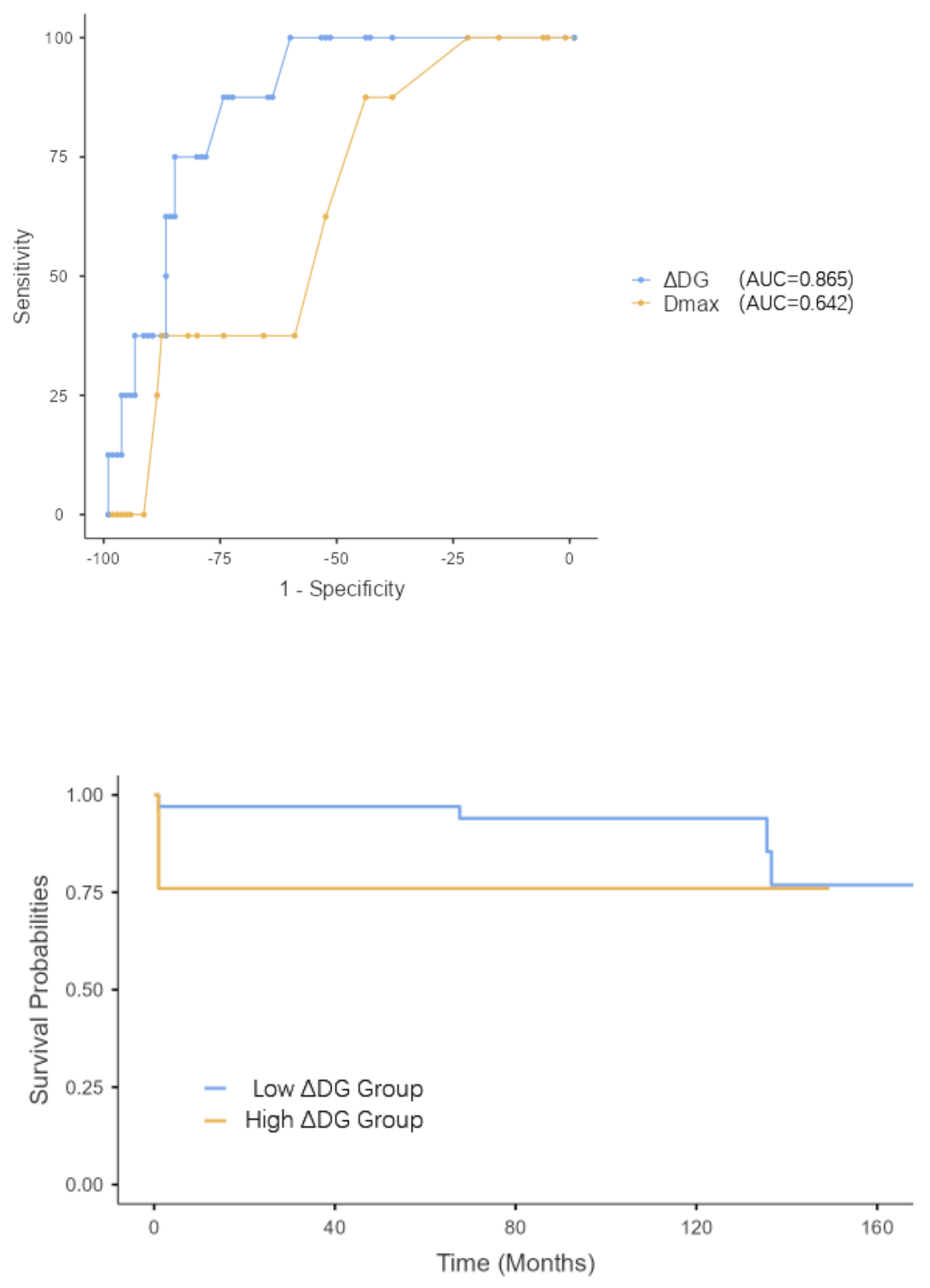